\title{
THE ROLE OF DRUGS IN NEONATAL RESUSCITATION
}

\author{
H.K. Muniraman, R. Roy
}

Neonatal Intensive Care Unit, Norfolk \& Norwich University Hospitals NHS Trust, Norwich, UK

Background: Approximately $10 \%$ of newborns require skilled neonatal resuscitation with airway and breathing management. Neonatal resuscitation drugs and cardiac compressions are used in less than $1 \%$ of deliveries. These infants have a poor prognosis and use of drugs in neonatal resuscitation remains one of the most controversial topics.

Objective: To document the outcome, in terms of morbidity and mortality in extreme and mature preterm's, term and near term infants receiving resuscitation drugs.

Methods: We retrospectively reviewed the records of all infants who received drugs during resuscitation between July 2006 and February 2011 from the neonatal database and follow up clinic letters.

Results: During the study period 26,881 infants were delivered of whom $17(0.06 \%)$ infants received resuscitation drugs and 69(0.25\%) infants received chest compressions. Overall $10(58 \%)$ infants survived, mortality was high $(50 \%)$ in infants born extreme preterm $(\leq 26$ weeks), infants born in asystole and in all infants receiving more than one dose of adrenaline. Significant neurodevelopmental disability was noted in surviving extreme preterm infants $(100 \%)$ compared to term infants $(25 \%)$. Abnormal cranial imaging was seen in $78 \%$ of all surviving preterm and in $62 \%$ of the term infants.

Conclusions: There is a role for resuscitation drugs in term, near term and mature preterm infants in restoring circulation in depressed neonates. In extremely preterm infants it is associated with significant mortality and neurodisability. Drugs should not be considered if adequate ventilation and cardiac compressions fail to stabilise extreme preterm infant. 